\title{
Stronger together: how to implement oncology and palliative care co-management
}

\author{
Juliet Jacobsen, MD, DPH, ${ }^{\mathrm{a}}$ Joseph Greer, PhD, Jennifer Temel, MD, ${ }^{\mathrm{c}}$ William Pirl, MD, ${ }^{\mathrm{d}}$ \\ and Vicki Jackson, MD, $\mathrm{MPH}^{\mathrm{a}}$
}

${ }^{a}$ Division of Palliative Care, ${ }^{b}$ Department of Psychiatry, ${ }^{c}$ Division of Oncology, Harvard Medical School, and Massachusetts General Hospital, Boston; and dDepartment of Psychiatry, University of Miami Health Systems, Florida

Outpatient palliative care is increasingly delivered through co-management, a collaborative model of care that enables palliative care clinicians and oncologists to coordinate efforts. Here, we offer a distillation of our experience with co-management at a large teaching hospital. We describe three strategies to implement co-management: a shared understanding of each subspecialty, a shared framework to help patients cultivate prognostic awareness, and a shared vision for the clinical goals. We hope that this synthesis will foster the development of co-management.

\begin{abstract}
A growing body of evidence shows that cancer patients benefit from early palliative care. ${ }^{1-4}$ As a result, key national oncology organizations have established referral standards for the provision of early palliative care. ${ }^{5,6}$ However, the optimal timing and intensity of early palliative care continues to be investigated. ${ }^{7-10}$ Early palliative care can be delivered through a variety of service models such as instructional sessions with or without monthly phone calls, "curbside" advice or only limited assistance during tumor board meetings, consultation, or co-management. ${ }^{1,11}$ In this paper, we describe our experience implementing co-management, the most comprehensive delivery model for early palliative care, and the model that most closely integrates palliative care and oncology.
\end{abstract}

\section{Co-management}

The Center to Advance Palliative Care Improving Outpatient Palliative Care Project has defined comanagement as occurring when the oncologist and the palliative care clinicians jointly make medical decisions and recommendations, unlike in traditional consultative care, in which the consultant offers advice, opinions, or recommendations to the oncologist who provides the ongoing care. Although overall responsibility for care remains with the oncologist, the palliative care clinician is often responsible for discrete care domains such as symptoms or advance care planning. ${ }^{12}$ Although early involvement of palliative care is associated with better outcomes, ${ }^{1}$ co-management can occur at any time in the disease trajectory and in the inpatient or outpatient setting. Successful co-management requires close collaboration between care providers and, in the outpatient setting, clinic co-localization with the palliative care clinic located within or near the oncology clinic. Ideally, co-management is provided by palliative care nurse practitioners or physicians in conjunction with the palliative care interdisciplinary team.

Co-management benefits patients and clinicians. For patients, co-management provides intensive palliative care. In the inpatient setting, patients are often seen by the palliative care team daily. In the outpatient setting, patients have routinely scheduled visits, often monthly. To minimize trips to the clinic, outpatient palliative care appointments are ideally scheduled on the same day as the patient's oncology appointments. This close follow-up allows palliative care clinicians to tightly manage symptom related problems and to engage in emotional work that facilitates coping, acceptance, and planning. ${ }^{13}$ One patient described her experience with co-management as follows,

\footnotetext{
... she [the palliative care clinician] is wonderful to talk to ... That has really made it more comfortable for me. You know, I look forward to these visits when I come down here. I can't always say I look forward to my doctors' visits, because I'm always nervous. But for these visits, I have no problem coming down. ${ }^{14}$
}

Accepted for publication November 2, 2016. Correspondence: Juliet Jacobsen, MD, DPH; Jjacobsen@partners.org. Disclosures: The authors report no disclosures or conflicts of interest. JCSO 2016;14(12):494-500. C2016 Frontline Medical Communications. doi: 10.12788/jicso.0310. 
Patients who experience early palliative care through co-management have a range of positive outcomes, which include improved symptom management, quality of life, mood, prognostic understanding, and survival, and less aggressive care at the end of life. ${ }^{3,8,15}$

Clinicians also benefit from co-management. Oncologists report unique and difficult challenges when caring for dying patients, including emotionally draining communication, an overwhelming responsibility for life and death, and the limitations of oncology to heal. ${ }^{16}$ Furthermore, oncologists who identify primarily with their biomedical role are at higher risk for feeling a sense of failure and an absence of collegial support. ${ }^{17}$ Both oncologists and palliative care clinicians are at high risk for burnout, ${ }^{18}$ a multidimensional construct that includes personal resources and work demands. ${ }^{19}$ In our experience, co-management can reduce burnout for palliative care and oncology clinicians by allowing them to partner in the care of complex patients. As an example, oncologists practicing in a cancer center with a routine practice of co-management reported feeling supported and that they relied on palliative care clinicians to "share the load."

\section{Clinician roles in co-management}

Patients and caregivers who experience co-management describe the roles of the oncologist and palliative care clinician as being discrete and complementary. On the one hand, they see oncology care as being "disease-centered," with the focus on disease control, cancer treatment, and survival, with the visits being more structured and led by the physician; and on the other hand, they see palliative care as being "more holistic and person-focused," where symptom management is a priority and the visits are more flexible and led by the patient. ${ }^{14}$

Clinicians who co-manage patients describe their roles with patients as overlapping yet distinct. Both oncologists and palliative care clinicians assess and manage symptoms and illness status. However, the palliative care clinician attends more to psychosocial elements, such as patient coping and cultivating illness understanding and prognostic awareness, whereas the oncologist focuses on cancer treatment and the management of medical complications. ${ }^{20}$ Palliative care clinicians report an additional role: interpreting the oncologist for the patient and the patient for the oncologist. For example, the palliative care clinician asks the patient about the oncology visit and then discusses information that was confusing or emotionally difficult. The palliative care clinician also provides feedback of patient perceptions to the oncologist, as one summed it up:

I will touch base with the oncologist to say, 'I am a little concerned about what [the patient] is understanding ${ }^{\prime} .{ }^{13}$

In this paper, we describe how oncologists and pallia- tive care clinicians can implement co-management. In our experience, co-management works most effectively if it is based on three strategies: a shared understanding of each subspecialty; a shared framework for how patients cultivate prognostic awareness; ${ }^{21}$ and a shared vision for the clinical goals. We will describe each strategy in turn.

\section{A shared understanding of each subspecialty}

Each clinician must learn enough about the other field to work with the role overlap in co-management. Palliative care clinicians should know enough basic oncology to manage symptoms and help patients process the prognosis and disease trajectory. Oncologists should know basic symptom management and communication skills and how to introduce the topic of palliative care to patients. To learn more about each specialty, clinicians can do occasional joint visits. The following section elaborates on these themes.

\section{Palliative care clinicians should know basic oncology}

Palliative care clinicians who co-manage oncology patients should know the indications and common side effects of standard cancer therapies in common diseases, such as lung, pancreatic, and colon cancer, and be ready to ask the oncologist follow-up questions. So for a patient receiving first-line treatment for non-small-cell lung cancer, the palliative care clinician should know whether the patient is receiving is standard therapy and, if not, why the oncologist recommended a different treatment. One reason could be that often oncologists will recommend milder therapy for patients with poor functional status. Palliative care clinicians should also be aware of how targeted therapies and immunotherapies are rapidly changing care and prognosis so that they can help patients calibrate their expectations.

\section{Oncologists should know how to introduce palliative care to patients}

When working with palliative care, oncologists must be able to explain palliative care to patients. Most members of the public do not have negative beliefs about palliative care. ${ }^{22}$ However, many advanced cancer patients initially perceive palliative care to be about death, hopelessness, dependency, and end of life, and attribute such perceptions to experiences in the health care system. ${ }^{23,24}$ Patients' initial misgivings often can be relieved if the oncologist explains the rationale for early palliative care. As one patient put it,

Well, when I hear palliative care ... naturally, I think, 'Oh God, I'm not long for the world.' But when she explained it to me ... I thought, 'well people should have thought of that before' ${ }^{23}$

A useful starting point is the standard definition of palliative care created by the Center to Advance Palliative Care, which worked with patients and families to determine the 
most acceptable phrasing. The center describes palliative care and the medical subspecialty of palliative medicine as specialized medical care for people living with serious illness. It focuses on providing relief from the symptoms and stress of a serious illness, whatever the diagnosis. The goal is to improve quality of life for both the patient and the family. Palliative care is provided by a team of palliative care doctors, nurses, and other specialists who work together with a patient's other doctors to provide an extra layer of support. It is appropriate at any age and at any stage in a serious illness and can be provided along with curative treatment. ${ }^{25}$

The explanation may need to be adjusted depending on the patient. For patients anxious about prognosis and end of life, a simple emphasis on quality of life and symptom management may suffice. Other patients may appreciate an in depth explanation.

\section{Both specialists should learn about the other specialty through joint visits}

Oncologists and palliative care clinicians can learn what the other does by occasionally seeing patients jointly. Joint visits can be planned, or serendipitous, when the clinicians happen to meet in the patient's room. Clinicians may spend some, or all, of the visit together with the patient, or may visit sequentially, where the first clinician gives a verbal handoff summarizing the visit to the second clinician while both clinicians are in the room.

Joint visits improve patient care in several ways. First, in our experience, patients appreciate seeing their clinicians working together to provide coordinated care. This is particularly true at times of transition, such as when scan results are back and we are making a new treatment plan. To the extent that joint visits improve oncologists' skill and comfort with end-of-life care, they also benefit future patients because oncologists who are more comfortable with end-of-life care have higher rates of specialist palliative care referral and primary palliative care delivery. ${ }^{26}$ Second, patient care is improved by joint visits because clinicians have additional support through difficult conversations with patients and families. ${ }^{13} \mathrm{~A}$ second clinician in the room can pick up subtle emotional cues or probe deeper when patients do not seem to understand. A second clinician can also help patients work through strong emotions so that there is less temptation for the clinician to retract their recommendations. As a colleague told us, "It helps to have you [palliative care] in the room with me so that I stick with my plan. I don't want to offer things in the moment that I don't really think will help. It is just so hard to respond to desperation when I am alone."

Joint visits can make clinical care more or less efficient. When clinicians visit sequentially, the second clinician can often save time by relying on the first clinician's clinical summary. Similarly, for complex discussions about goals of care, our experience is that discussions are often more effective when both clinicians are in the room. And more generally, family meetings that include palliative care have been found to be effective in helping patients and families meet their care needs. ${ }^{27,28}$ Joint visits can be less efficient when each clinician engages in a specialty-specific discussion. Even so, information from these discussions is often valuable for patient care. For example, observing the chemotherapy consent process can help the palliative care clinician develop insight into the patient's expectations for treatment. Similarly, observing an in depth coping assessment can help the oncologist develop insight into the patient's quality of life. When joint visits are not feasible due to constraints on time, clinical space, or scheduling, clinicians can support each other by communicating regularly by phone, e-mail, or in person.

\section{Both clinicians should recognize that patients sometimes tell different things to different clinicians}

When working together, both clinicians should understand that a common phenomenon in co-management is discrepant patient reports: when patients say one thing to their oncologist and another thing to the palliative care clinician. $^{29}$

Patients give discrepant reports for several reasons. Many patients feel safe while being treated with chemotherapy and worry what will happen when treatments run out. Even fatigued or in pain, patients worry about the oncologist "giving up" on them by reducing, delaying, or stopping treatment. ${ }^{16,30}$ Patients may therefore tell their oncologist that they feel well and, on the same day, tell their palliative care clinician that they feel poorly. In addition, patients worry about disappointing their oncologist, with whom they have closely bonded after years of treatment. With these strong ties and the desire to be a "good" patient, patients may hesitate to discuss new symptoms. In one case, a patient called her palliative care clinician to report new onset dyspnea. When asked why she did not call the oncologist, the patient replied the oncologist had been working so hard that she "did not want to upset her."

With co-management, patients can confide in the palliative care clinician without worrying about what the oncologist might think. Then, the palliative care clinician can work with the patient and the oncologist to figure out next steps. As one patient stated,

I see the palliative care doctor's role as being more supportive emotionally as well as physically. They're not into planning your treatment, that's the big difference ... It's more of a supportive role, but also certainly keeping tabs on how I am doing physically so she can support me. ${ }^{14}$ 


\section{A shared framework for how patients cultivate prognostic awareness}

We define prognostic awareness as developing a deeper understanding of the illness trajectory and/or timeline. The framework for how patients cultivate prognostic awareness explains how patients balance their needs for hope and for prognostic information, and why patients who have been told the prognosis sometimes seem not to understand it. Using the framework, oncology and palliative care clinicians can help patients cultivate prognostic awareness and make informed medical decisions. In the following paragraphs, we describe the framework and its benefits.

The essential idea is that patients develop prognostic awareness by swinging, like a pendulum, between moments of realism, when they can acknowledge the prognosis, and moments of optimistic hope, when they seem to not understand the prognosis (see diagram). ${ }^{21,31-33}$ Swinging toward optimistic hope is healthy and normal and helps patients modulate the strong emotions that accompany a serious prognosis. ${ }^{34}$ Swinging toward a realistic perspective helps patients plan for the future and set expectations for treatment. For example, one patient described the experience as follows:

\section{It's been hard since I heard the bad news about my dis- ease. Sometimes I feel very hopeful and think positively about the future. Other times, I feel fearful and sad because I know how serious my illness is. I seem to go back and forth between those two feelings. ${ }^{33}$}

In our experience, when clinicians are unaware of the swinging pendulum framework, they misinterpret statements of optimistic hope. For example, the palliative care clinician might assume that a patient with metastatic cancer who makes hopeful statements about cure has not been told the true prognosis and blame the oncologist for not being honest. Or either clinician might misinterpret optimistic hope as evidence of denial, as a sign that the patient cannot cope with the prognosis. Both clinicians may resist supporting or even tolerating optimistic hope lest the support be misleading. Framing optimistic hope within a healthy dialectic, in which optimistic hope and realism coexist, helps prevent these misunderstandings.

\section{Benefits of the swinging pendulum framework}

By sharing the swinging pendulum framework, palliative care and oncology clinicians can support optimistic hope. Although clinicians should agree only with patients' true hopeful statements, they can empathize with the wish that things were different: "I wish that that there were a cure for this terrible disease." 35 Such "I wish" statements are a powerful way to empathize without being misleading. Clinicians can also gently help patients calibrate expectations by supporting more attainable hopes, by saying things such as: "I would love to see you make it to your daugh-

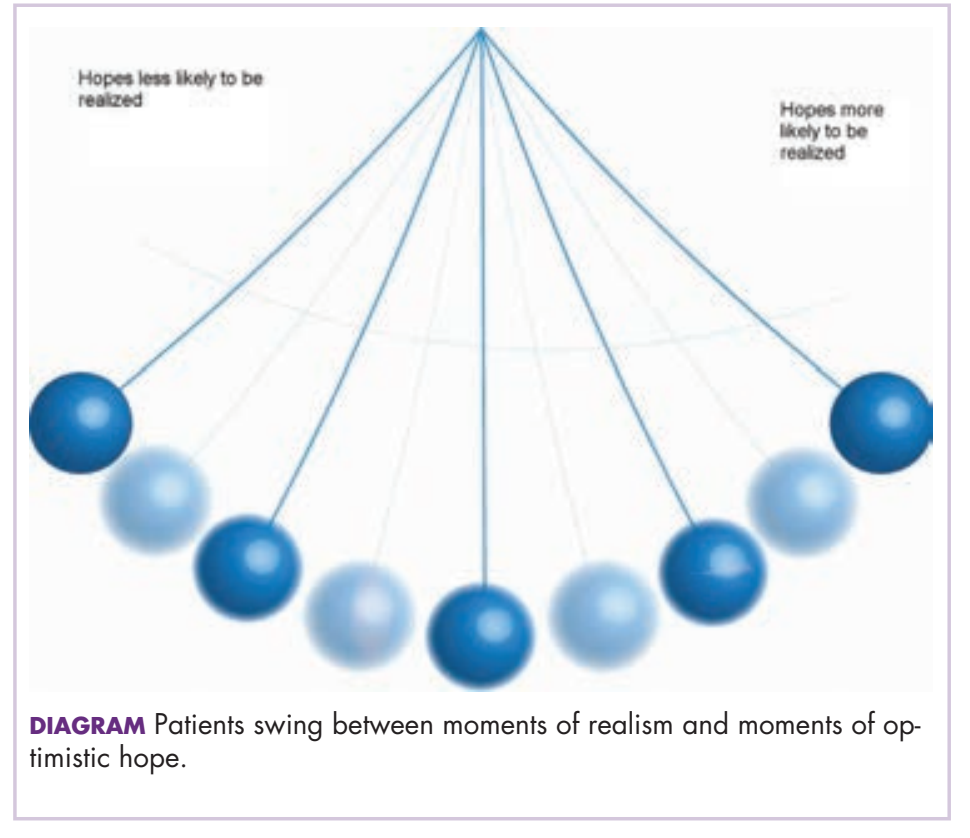

ter's graduation next year. I think that is something we can hope for." In clinically stable situations, when no medical decision making is needed, clinicians can support optimistic hope and allow patients time to cope with their illness before more explicitly discussing the prognosis.

The framework also helps oncology and palliative care clinicians recognize strained patient coping that may reduce a patient's desire for prognostic information. When patients move back and forth frequently between optimistic hopes and realism (and cannot focus on both at the same time), they tend to view prognostic information as a threat to hope and prefer that clinicians provide prognostic information cautiously and indirectly. ${ }^{33}$ Furthermore, in our experience, when patients express only hope and do not swing to a realistic perspective, this one-sidedness may reflect difficulty coping or untreated depression that also reduces the patient's desire for prognostic information and ability to integrate it.

\section{How to work together to cultivate prognostic awareness}

Both clinicians can help patients cultivate prognostic awareness. Clinicians may visit jointly to assess how the patient is coping with the illness and explore the patient's hopes, goals, and worries should s/he become sicker. Or, the palliative care clinician may use ever more graduated discussions that gently help the patient imagine the future and adapt to the reality that time is shorter than was hoped. ${ }^{21}$

How deeply to discuss prognosis will depend on the clinical urgency and the patient's information preferences. Very sick patients require urgent discussion. For stable patients who are ambivalent about prognostic information, these discussions can deepen as the patient learns to manage the resulting strong emotions. For a small number of patients, particularly those who are young or who struggle to cope, 
TABLE A sample documentation template for prognostic awareness

\begin{tabular}{|c|c|}
\hline What is being addressed & How the information is understood \\
\hline Patient's understanding of illness & $\begin{array}{l}\text { Jane understands that her cancer is not curable. She knows that it has spread to her } \\
\text { bones and to her brain. }\end{array}$ \\
\hline Family's understanding of illness & Jane's husband Bob also understands that the disease is not curable. \\
\hline Hopes/goals & $\begin{array}{l}\text { Jane is hoping for a cure through a breakthrough in targeted therapy. She is also hoping } \\
\text { to have as much time with her children as possible. }\end{array}$ \\
\hline Fears/worries & Jane worries about how her children will manage if she dies. \\
\hline Estimated prognosis & $1-2$ years \\
\hline Prognosis discussed with patient? & $\begin{array}{l}\text { Dr } O \text { has told Jane that the cancer was not curable. They have not discussed a time- } \\
\text { based or functional prognosis. }\end{array}$ \\
\hline Patient's response & $\begin{array}{l}\text { Jane was very upset when told the cancer was not curable and further discussion about } \\
\text { how much time to expect was postponed. }\end{array}$ \\
\hline
\end{tabular}

too much explicit discussion of prognosis is not helpful and, in our experience, is not necessary for a "good death." These patients may feel overwhelmed and instead choose minimal discussion with just enough big-picture information to make decisions, or they may transfer decision making to a family member. ${ }^{36}$ Through ongoing discussions, both clinicians can work with the patient to determine how much prognostic information is needed for informed medical decision making.

We hypothesize that the trusting relationship that patients develop with their palliative care clinician through co-management is therapeutic and promotes the cultivation of prognostic awareness. The safe relationship enables patients to explore and process their fears related to the prognosis and it leads to greater acceptance. Rather than challenging a patient's optimistic hopes, the palliative care clinician aims to prepare patients for medical decision making by facilitating adaptation. Patients who receive early palliative care through co-management retain or develop a more accurate perception of their prognosis and are less likely to receive intravenous chemotherapy near the end of life. ${ }^{15}$ One patient described the cultivation of prognostic awareness as follows,

That's the progress of the illness. I think you start there [back and forth] the minute you hear [the diagnosis] and you hope for a fix. And with the progress of the illness, you hope for ... other things, for a gentle death. ${ }^{33}$

By frequently discussing the patient's development of prognostic awareness using the shared framework, oncology and palliative care clinicians can maintain a unified message to the patient. The palliative care clinician should tell the oncologist what the patient understands about the goals of treatment and how the patient is integrating prognostic information. This information can help the oncologist plan further discussions, particularly with a struggling patient. Because discussions with the patient about prognosis are revisited over the course of disease, ongoing dis- cussion between the clinicians allows the clinicians to compare experiences to identify when the patient is struggling, and to recognize that, even with skilled and explicit communication about prognosis, the patient may not integrate the information. A patient's misunderstanding of the prognosis does not necessarily indicate that information wasn't communicated well. To document a patient's development of prognostic awareness, we suggest a structured approach so that all members of the care team know what the patient has been told and how the patient is integrating this information (Table). Ideally, such a template would be located centrally in the medical record, so that all clinicians can contribute to documenting prognostic awareness.

\section{A shared vision for the clinical goals}

The final co-management strategy is sharing a vision for the clinical goals. The goal may be cure, prolonged survival, better symptom control (perhaps with cancer-directed therapy), or a combination. Sometimes, the goal may be a reach. If the palliative care clinician is not clear about the goal, s/he should ask the oncologist for clarification. After the goal has been established, both clinicians can define and parcel out the resulting tasks, such as determining any appropriate disease-modifying therapy; providing pain and symptom management; managing medical complications of the disease and treatment; cultivating patients' prognostic awareness; and supporting one another. Explicit discussion of who does which task or tasks will streamline care delivery. Both clinicians should aim to give consistent prognostic information and then support the patient as s/ he develops a deeper understanding and tolerance of this reality. By balancing reality with hope, both clinicians also model for the patient how to cope and live well despite the prognosis.

In our experience, co-management is most effective when the oncologist "steps back"11 and asks the palliative care clinician to take a central role in symptom control. For example, the oncologist might say to the patient, "I am going to 
ask palliative care to work on your pain management. They are the experts." Collaborating with palliative care clinicians on particular symptoms can give the oncologist time with the patient to discuss treatment options and to manage complications from the cancer and its treatment. Being explicit about the role of palliative care also tells the patient that the oncologist and palliative care clinicians are collaborating. Palliative care clinicians can then develop rapport and trust with the patient through symptom assessment and treatment. By helping patients feel better, the palliative care clinician develops a helping, nonthreatening role. ${ }^{13}$ Once trust has been established, the more difficult conversations about illness understanding and prognostic awareness deepen over time. ${ }^{37}$

\section{References}

1. Davis MP, Temel JS, Balboni T, Glare P. A review of the trials which examine early integration of outpatient and home palliative care for patients with serious illnesses. Ann Palliat Med. 2015;4(3):99-121.

2. El-Jawahri A, Greer JA, Temel JS. Does palliative care improve outcomes for patients with incurable illness? A review of the evidence. J Support Oncol. 2011;9(3):87-94.

3. Temel JS, Greer JA, Muzikansky A, et al. Early palliative care for patients with metastatic non-small-cell lung cancer. N Engl J Med. 2010;363(8):733-42.

4. Maloney C, Lyons KD, Li Z, Hegel M, Ahles TA, Bakitas M. Patient perspectives on participation in the ENABLE II randomized controlled trial of a concurrent oncology palliative care intervention: benefits and burdens. Palliat Med. 2013;27(4):375-383.

5. Levy M, Smith T, Alvarez-Perez A, et al. Palliative Care Version 1.2016. J Natl Compr Canc Netw. 2016;14(1):82-113.

6. Smith TJ, Temin S, Alesi ER, et al. American Society of Clinical Oncology provisional clinical opinion: the integration of palliative care into standard oncology care. J Clin Oncol. 2012;30(8):880-887.

7. Bakitas MA, Tosteson TD, Li Z, et al. Early versus delayed initiation of concurrent palliative oncology care: patient outcomes in the ENABLE III randomized controlled trial. J Clin Oncol. 2015;33(13):1438-1445.

8. Zimmermann C, Swami N, Krzyzanowska M, et al. Early palliative care for patients with advanced cancer: a cluster-randomised controlled trial. Lancet. 2014;383(9930):1721-1730.

9. Bakitas M, Lyons KD, Hegel MT, et al. Effects of a palliative care intervention on clinical outcomes in patients with advanced cancer: the Project ENABLE II randomized controlled trial. JAMA 2009;302(7):741-749.

10. Lobb EA, Lacey J, Kearsley J, Liauw W, White L, Hosie A. Living with advanced cancer and an uncertain disease trajectory: an emerging patient population in palliative care? BMJ Support Palliat Care. 2015;5(4):352-357.

11. Bakitas M, Lyons KD, Hegel MT, Ahles T. Oncologists' perspectives on concurrent palliative care in a National Cancer Institutedesignated comprehensive cancer center. Palliat Support Care. 2013;11(5):415-423.

12. Barbour LT, Cohen SE, Jackson VA, et al. Models for palliative care outside the hospital setting: a technical assistance monograph from the IPAL-OP ProjectThe IPAP-OP Project, Center to Advance Palliative Care; 2012. https://central.capc.org/eco_download. php?id=1130 [password protected]. Dated 2012. Accessed November 29, 2016.

13. Back AL, Park ER, Greer JA, et al. Clinician roles in early integrated palliative care for patients with advanced cancer: a qualitative study. J Palliat Med. 2014;17(11):1244-1248.

14. Hannon B, Swami N, Pope A, et al. Early Palliative care and its role in oncology: a qualitative study. Oncologist. 2016;21:1387-1395.

15. Temel JS, Greer JA, Admane S, et al. Longitudinal perceptions of prognosis and goals of therapy in patients with metastatic non-smallcell lung cancer: results of a randomized study of early palliative care.

\section{Conclusion}

Co-management allows clinicians to deliver comprehensive care to patients with advanced cancer. It requires a strong partnership with a shared understanding of each subspecialty, a shared framework for how patients cultivate prognostic awareness, and a shared vision for the clinical goals. Co-management works most effectively when clinicians provide a consistent message to the patient and are grateful for the complementary skills and roles of each subspecialty. Through co-management, even in the most difficult clinical circumstances, palliative care and oncology clinicians can provide patients and families with high quality care.

J Clin Oncol. 2011;29(17):2319-2326.

16. Bluhm M, Connell CM, De Vries RG, Janz NK, Bickel KE, Silveira MJ. Paradox of prescribing late chemotherapy: oncologists explain. http://ascopubs.org/doi/full/10.1200/JOP.2016.013995. Published September 20, 2016. Accessed October 30, 2016.

17. Jackson VA, Mack J, Matsuyama R, et al. A qualitative study of oncologists' approaches to end-of-life care. J Palliat Med. 2008;11(6):893-906.

18. Kearney MK, Weininger RB, Vachon ML, Harrison RL, Mount BM. Self-care of physicians caring for patients at the end of life: 'Being connected ... a key to my survival.' JAMA. 2009;301(11):1155-1164, E1.

19. Back AL, Steinhauser KE, Kamal AH, Jackson VA. Building resilience for palliative care clinicians: an approach to burnout prevention based on individual skills and workplace factors. J Pain Symptom Manage. 2016;52(2):284-291.

20. Yoong J, Park ER, Greer JA, et al. Early palliative care in advanced lung cancer: a qualitative study. JAMA Intern Med. 2013;173(4):283-290.

21. Jackson VA, Jacobsen J, Greer JA, Pirl WF, Temel JS, Back AL. The cultivation of prognostic awareness through the provision of early palliative care in the ambulatory setting: a communication guide. $J$ Palliat Med. 2013;16(8):894-900.

22. 2011 Public Opinion Research on Palliative Care: A report based on research by public opinion strategies [Internet]. https://central.capc. org/eco_download.php?id=311 [password protected]. Dated 2011. Accessed November 29, 2016.

23. Zimmermann C, Swami N, Krzyzanowska M, et al. Perceptions of palliative care among patients with advanced cancer and their caregivers. CMAJ. 2016;188(10):E217-227.

24. Horlait M, Chambaere K, Pardon K, Deliens L, Van Belle S. What are the barriers faced by medical oncologists in initiating discussion of palliative care? A qualitative study in Flanders, Belgium. Support Care Cancer. 2016;24(9):3873-3881.

25. About palliative care [Internet]. https://www.capc.org/about/palliative-care/. Accessed October 1, 2016

26. Hui D, Cerana MA, Park M, Hess K, Bruera E. Impact of oncologists' attitudes toward end-of-life care on patients' access to palliative care. Oncologist. 2016;21(9):1149-1155.

27. Hudson P, Thomas T, Quinn K, Aranda S. Family meetings in palliative care: are they effective? Palliat Med. 2009;23(2):150-157.

28. Hannon B, O'Reilly V, Bennett K, Breen K, Lawlor PG. Meeting the family: measuring effectiveness of family meetings in a specialist inpatient palliative care unit. Palliat Support Care. 2012;10(1):43-49.

29. Jacobsen J, Thomas JD, Jackson VA. Misunderstandings about prognosis: an approach for palliative care consultants when the patient does not seem to understand what was said. J Palliat Med. 2013;16(1):91-95.

30. Martinsson L, Axelsson B, Melin-Johansson C. Patients' perspectives on information from physicians during palliative chemotherapy: a qualitative study. Palliat Support Care. 2016;14(5):495-502. 


\section{How We Do It}

31. Jacobsen J, Jackson VA. A communication approach for oncologists: understanding patient coping and communicating about bad news, palliative care, and hospice. J Natl Compr Canc Netw. 2009;7(4):475-480.

32. Weisman A. On dying and denying. New York, NY: Behavioral Publications Inc; 1972.

33. Curtis JR, Engelberg R, Young JP, et al. An approach to understanding the interaction of hope and desire for explicit prognostic information among individuals with severe chronic obstructive pulmonary disease or advanced cancer. J Palliat Med. 2008;11(4):610-620.

34. Block SD. Psychological issues in end-of-life care. J Palliat Med.
2006;9(3):751-772.

35. Quill TE, Arnold RM, Platt F. 'I wish things were different': expressing wishes in response to loss, futility, and unrealistic hopes. Ann Intern Med. 2001;135(7):551-555.

36. Back AL, Arnold RM. Discussing prognosis: 'How much do you want to know?'Talking to patients who are prepared for explicit information. J Clin Oncol. 2006;24(25):4209-4213.

37. Jacobsen J, Kvale E, Rabow M, et al. Helping patients with serious illness live well through the promotion of adaptive coping: a report from the improving outpatient palliative care (IPAL-OP) initiative. J Palliat Med. 2014;17(4):463-468. 